\title{
Attitudes of radiation oncologists toward palliative and supportive care in the United States: Report on national membership survey by the American Society for Radiation Oncology (ASTRO)
}

\author{
Randy L. Wei, MD, PhD ${ }^{a},{ }^{,}$, Malcolm D. Mattes, MD $^{b}$, James $\mathrm{Yu}, \mathrm{MD}^{\mathrm{c}}$, Adrienne Thrasher, \\ MHS ${ }^{d}$, Hui-Kuo Shu, MD ${ }^{e}$, Harald Paganetti, PhD $^{f}$, Jennifer De Los Santos, MDg, Bridget \\ Koontz, MD ${ }^{h}$, Christopher Abraham, MD', and Tracy Balboni, MDj \\ aDepartment of Radiation Oncology, University of California, Irvine, California

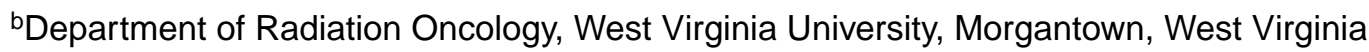 \\ 'Department of Radiation Oncology, Yale School of Medicine, New Haven, Connecticut \\ dAmerican Society for Radiation Oncology, Arlington, Virginia \\ eDepartment of Radiation Oncology, Emory School of Medicine, Atlanta, Georgia \\ fDepartment of Radiation Oncology, Massachusetts General Hospital \& Harvard Medical School, \\ Boston, Massachusetts \\ 9Department of Radiation Oncology, University of Alabama, Birmingham, Alabama \\ hDepartment of Radiation Oncology, Duke University, Durham, North Carolina \\ 'Department of Radiation Oncology, Washington University in St. Louis, St. Louis, Missouri \\ 'Department of Radiation Oncology, Dana-Farber/Brigham and Women's Cancer Center, Boston, \\ Massachusetts
}

\section{Abstract}

Purpose-Radiation oncologists are frequently involved in providing palliative and supportive care (PSC) for patients with advanced cancers through delivery of palliative radiation. Whether they are confident in their ability to assess and initiate treatments for pain, nonpain, and psychosocial distress is unknown. The American Society for Radiation Oncology surveyed its practicing members in the United States on self-assessment of their primary PSC skills and access to continuing medical education on PSC.

Methods-We electronically surveyed 4093 practicing radiation oncologists in the United States. The survey consisted of 16 -questions in 5 sections ${ }^{1}$ : demographics, ${ }^{2}$ PSC training, ${ }^{3}$ domains of PSC, ${ }^{4}$ perceived barriers as a radiation oncologist to initiate advanced care planning, and ${ }^{5}$ discussion of prognosis.

*Corresponding author. Department of Radiation Oncology, University of California, Irvine, 101 The City Drive, Orange, CA 92868. rwei@uci.edu (R.L. Wei).

Supplementary material for this article (http://dx.doi.org/10.1016/j.prro.2016.08.017) can be found at www.practicalradonc.org. 
Results-The survey was e-mailed to 4093 American Society for Radiation Oncology members, and 649 responses were received (response rate 16\%). The majority (91\%) of radiation oncologists surveyed believe PSC is an important competency for radiation oncologists. Most radiation oncologists reported that they are moderately confident in their ability to assess and manage pain and gastrointestinal symptoms, but less confident in their ability to manage anorexia, anxiety, and depression. Despite areas of decreased confidence, a large number (42\%) of radiation oncologists do not receive any additional PSC education beyond their residency training. Lastly, a perceived fear of upsetting referring medical oncologists and lack of clinic time are concerns for radiation oncologists who may want to initiate goals of care/advance care planning discussions with patients and their families.

Conclusion-Radiation oncologists are more confident in their ability to assess and manage pain than in their ability to manage depression, anxiety, anorexia, and fatigue. There is a need for increasing continuing medical educational efforts in PSC for practicing radiation oncologists, and strengthening PSC training in residency programs.

\section{Introduction}

Palliative and supportive care (PSC) is a discipline focused on optimizing the quality of life — physical, emotional, social, and spiritual—of patients and their families in the setting of serious illnesses, including cancer. Increasing numbers of new cancer diagnoses coupled with advances in medicine have led to longer survival in patients with advanced stage disease and have resulted in a growing demand for and a shortage of PSC specialists. ${ }^{1}$ Thus, oncologists play a greater role in providing generalist PSC that includes basic management of pain and symptoms, psychosocial distress, and communication skills when discussing prognosis and goals of care. ${ }^{2}$

Radiation oncologists are frequently involved in providing care to patients who face advanced cancers given the utility of palliative radiation therapy for controlling regions of disease causing symptoms and/or compromising function. In fact, data indicate that approximately $30 \%$ to $50 \%$ of radiation oncology treatment courses are palliative in intent, highlighting the need for radiation oncologists to be competent in providing generalist PSC to patients with advanced cancer. ${ }^{3-5}$ Patients receiving palliative radiation commonly experience depression, anxiety, and psychosocial distress. ${ }^{6,7}$ Early identification and intervention, including referral to specialists, significantly affect patient quality of life and cancer-related fatigue..$^{8,9}$

To date, there has only been one published survey of oncologists by the European Society of Medical Oncology and one survey of pediatric oncologists by the American Society of Clinical Oncology on their role in palliative care. There has not been a comprehensive assessment of practicing oncologists, let alone radiation oncologists, in PSC in the United States. To identify areas for practice improvement, the American Society for Radiation Oncology (ASTRO) surveyed its practicing members in the United States on self-assessment of their primary PSC skills, access to continuing medical education on PSC, and perceived barriers to having a greater role in advance care planning and discussion on prognosis. 


\section{Methods and materials}

\section{Survey development}

The questions used in this electronic survey were adapted from a survey developed previously for radiation oncology residents to assess quality of training and perceived competency in PSC skills. ${ }^{10}$ The ASTRO working group modified items to adapt them to the population being surveyed-practicing radiation oncologists. The final survey tool was reviewed and approved by the Board of Directors of ASTRO. The survey consisted of 16 questions in 5 areas $^{1}$ : demographics ${ }^{2}$; PSC training ${ }^{3}$; domains of PSC $^{4}$; perceived barriers as a radiation oncologist to initiate advanced care planning; and ${ }^{5}$ discussion on prognosis (Supplementary Table 1). Within the domains of PSC, respondents were asked to rate their confidence using a Likert scale ( 1 = "Not at all confident"; 2 = "Minimally confident"; 3 = "Somewhat confident"; 4 = "Moderately confident"; and 5 = "Very confident") in providing specific skills that are required as part of primary PSC practice.

\section{Subjects}

The electronic survey was created and delivered via e-mail using commercial software (Qualtrics, Provo, UT). Usability and technical functionality of the electronic survey was tested before it was sent to all active radiation oncologists in the United States as of August 1, 2015, per the ASTRO membership database. Radiation oncologists who were not actively practicing, physicists, nurses, and radiation oncology residents-in-training were ineligible to participate. Physicians were not compensated for their participation. Four e-mail reminders were sent from October to November 2015 to those who had not yet responded. Only fully completed individual questions for a given respondent were included in the analysis of that question. All respondents had a unique survey link to ensure that no duplicate entries were recorded.

\section{Statistical analysis}

Only completed surveys were analyzed. Descriptive (summary) statistics are reported for the entire population of participants, with data presented as frequency counts, percentages of respondents who responded "Moderately confident" and "Very confident," medians, and means (with standard deviation), as indicated. Statistical analysis was performed using IBM SPSS Statistics version 22.0 (IBM Corp., Armonk, NY).

\section{Results}

\section{Demographics and previous training}

The survey was e-mailed to 4093 ASTRO members, and 649 responses were received (response rate 16\%). The demographics of these respondents are shown in Table 1. The most common response in each category was as follows: male (67\%), in practice for more than 21 years (34\%), in private/community practice (52\%), and from the Northeastern United States (28\%). The survey respondents are generally representative of the ASTRO membership, of whom $74 \%$ are male and $24 \%$ are from the Northeastern United States. 
Fifty-eight percent of respondents had received some form of PSC training after their residency. The most common types of additional training included institutional grand rounds/seminars (28\%), courses at the ASTRO annual meeting (24\%), online webinars (18\%), courses at other meetings/conferences (18\%), courses at the ASTRO spring refresher meeting (12\%), or involvement in a fellowship in palliative medicine (2\%). The respondents' years in practice did not correlate with the amount of palliative care training received beyond residency education $\left(\mathrm{r}_{\mathrm{s}}=0.033, P>.05\right)$. A total of 36 respondents $(5.5 \%)$, half of whom had been in practice for over 21 years, were dual board certified in palliative care and radiation oncology.

\section{Role of radiation oncologists in PSC}

Seventy-nine percent of respondents have a palliative medicine service at their institution, and $21 \%$ of them participate in palliative medicine rounds. Fewer private/community practice radiation oncologists (69\%) than academic/university radiation oncologists (92\%) reported having a palliative medicine team at their institution. Ninety-one percent of respondents either agreed or strongly agreed that palliative cancer care is an important competency for radiation oncologists, whereas only $2 \%$ were neutral or disagreed. Perceived barriers to discussing goals of care and advance care planning with patients included not wanting to upset the medical oncologist (31\%), inability to schedule clinic time to hold these conversations (31\%), poor insurance reimbursement (12\%), lack of interest in this clinical area (9\%), and other causes (17\%). Other causes that were manually entered by respondents included that these discussions are deferred to or managed by other physicians $(6 \%)$, issues with family and patient readiness/denial/expectations (4\%), and lack of training/knowledge (3\%).

\section{Self-assessment of competence in PSC}

Respondents' perceived levels of confidence in various aspects of pain assessment and management for an advanced cancer patient are shown in Table 2 and Supplementary Table 2. Respondents were most likely to report moderate or high confidence in their ability to assess pain (95.7\%). They were also more confident in managing somatic pain $(91.6 \%)$ than neuropathic pain $(69.1 \%)$ or opioid-related side effects $(68.5 \%)$. Respondents rated their abilities to perform opioid conversion and to rotate opioids as being the areas in which they were least confident (56.4\%). When asked about their level of confidence in caring for other nonpain symptoms, respondents were most confident in their ability to manage gastrointestinal symptoms $(82.8 \%)$. They were least confident in their ability to manage insomnia (44.3\%), anorexia (43.6\%), or fatigue (40.6\%) (Table 3 and Supplementary Table $3)$.

Respondents' perceived confidence in their ability to perform psychological, cultural, and spiritual aspects of care for a patient with advanced cancer is shown in Table 4 and Supplementary Table 4 . Respondents were more confident in recognizing when to incorporate interpreter services (82.4\%) and the importance of culture (80.4\%), and less confident in the initial management of anxiety (49.3\%) and depression (33.7\%). Additionally, when asked about their confidence in their ability to perform social, ethical, and legal aspects of care for a patient with advanced cancer, respondents were most 
confident in discussing the transition from curative to palliative care (82.8\%) and least confident in managing conflict between the patient/family and the clinical team (52.8\%) (Table 5 and Supplementary Table 5). Furthermore, in terms of their confidence in various aspects of advance care planning, respondents were most confident in their ability to communicate bad news (92.1\%) and discuss prognosis (85.8\%), and least comfortable discussing preparation of a living will (56.5\%) or do-not-resuscitate orders (65.1\%) (Table 6 and Supplementary Table 6).

Tables 2 through 6 and Supplementary Tables 2 through 6 also describe the differences in the percentage of "Moderately confident" and "Very confident" responses between subgroups of respondents who work in an academic practice versus a private/community practice. The private/community practice physicians consistently rated their confidence level in their PSC competency higher than academic physicians did, with statistically significant differences for certain questions, as indicated in the tables. Additionally, means, medians, and standard deviations of Likert ratings were calculated for all questions (Supplementary Table 1).

\section{Discussion}

Given the critical role that radiation oncologists play in modern cancer care, understanding the current attitudes and evolving role of radiation oncologists in PSC is important to identify areas of strength or need. This is the first published nationwide survey on the topic of PSC of practicing radiation oncologists in the United States. Surveys on PSC have been done for smaller groups of practicing physicians and medical residents/fellows in training, but surveys have never addressed a large group of physicians at a national level. ${ }^{11-18}$ The great majority $(91 \%)$ of radiation oncologists surveyed believe PSC is an important competency for radiation oncologists. Most radiation oncologists reported that they are moderately confident in their ability to assess and manage pain and gastrointestinal symptoms but less confident in their ability to switch a patient's opioid to another formulation to improve analgesic therapy, anorexia, anxiety, and depression. Lastly, a perceived fear of upsetting referring medical oncologists and lack of clinic time are concerns for radiation oncologists who may want to initiate goals of care/advance care planning discussions with patients and their families.

Confidence is uneven across the domains of PSC. Radiation oncologists were most confident in their ability to assess and treat pain, whereas they were less confident in prescribing alternative opioids and in managing opioid related side effects. Radiation oncologists often treat patients with palliative radiotherapy for painful metastatic and advanced cancers, and patients' pain must be well controlled if they are to tolerate their radiation treatments and optimize quality of life until palliative radiation reaches its full efficacy, a process that can take 2 to 3 weeks. ${ }^{19}$ Studies have shown that patients referred for palliative radiotherapy for symptomatic bone metastases are often not taking analgesics or are receiving weak opioids or non-opioids, ${ }^{20,21}$ thus making opioid management a critical skill set for radiation oncologists. This skill set is necessarily one that allows flexibility in prescribing between alternative routes and potencies to achieve analgesia across a broad range of clinical scenarios. ${ }^{22}$ Additionally, radiation oncologists reported less confidence in management of 
depression, anxiety, fatigue, anorexia, and insomnia. Treating these symptoms is important for a patient's ability to cope with physical pain, endure side effects related to disease or therapy, and maintain quality of life. ${ }^{23}$ Thus, radiation oncologists may benefit from guidelines delineating basic expectations regarding primary PSC skills to be practiced.

A large number (42\%) of respondents reported no further PSC education after residency, despite advances in PSC clinical research and evidence-based practice guidelines published by medical societies. ${ }^{24,25}$ Other respondents received continuing medical education in PSC from national meetings, including ASTRO, institutional seminars, and online webinars. However, because respondents can select multiple options, the actual number of individuals who attend such continuing medical education activities may be less. Interactive continuing medical education sessions that enhance participant activity and provide opportunities to practice skills in a simulated setting have a more significant influence on professional practice than didactic sessions. ${ }^{26}$ Ideally, physicians would have received such education during their residency training. There are currently no published studies on PSC education in radiation oncology training programs in the United States to assess the quality and quantity of formal PSC education. However, surveys of hematology/oncology fellows reported a lack of formal didactics in management of depression, opioid rotation, and communications skills, ${ }^{18}$ which is similar to the decreased confidence in these areas in our current survey.

Our survey sought to identify perceived barriers that limit radiation oncologists from initiating discussions on prognosis/goals of care and advance directives. A substantial minority of respondents indicated that they fear upsetting the referring medical oncologist $(31 \%)$. However, discussing prognosis and goals of care are vital to making personalized treatment recommendations. Additionally, current guidelines including the ACR-ASTRO practice parameter for communicationm recommend that goals of treatment intent (curative vs palliative) be listed in consult notes. Finally, discussions of advance care directives during a timeframe when a patient is still independent are important care issues that could remain unaddressed unless barriers and their solutions are defined.

Additionally, our survey showed that $12 \%$ of respondents indicate poor insurance reimbursement as a barrier against PSC conversations. In 2015, the Centers for Medicare \& Medicaid Services authorized the establishment and valuation of a code for advance care planning; however, there is currently no reimbursement. If the Centers for Medicare \& Medicaid Services finalize payment rates for these services, it would allow reimbursement of physician time to hold early conversations on goals of care both before the illness progresses and during the course of treatment to decide what type of care is in line with the patient's goals. ${ }^{27}$ Thus, practicing radiation oncologists would benefit from additional training in PSC such that discussion and management of palliative care would be a billable charge.

Community physicians rated higher confidence in all PSC domains than their academic counterparts. In total, $79 \%$ of respondents have access to palliative care teams within their hospital or group practice (69\% of community radiation oncologists and $92 \%$ of academic radiation oncologists). Greater confidence on the part of community physicians may be due to the fact that more limited access to palliative care teams encourages radiation oncologists 
to fulfill greater PSC roles and build their PSC competencies. Additionally, the increased exposure to palliative care teams on the part of academic physicians may heighten awareness of the complexities of specialty palliative care, resulting in greater appreciation of their PSC deficiencies.

Furthermore, our findings demonstrated that $79 \%$ of respondents have access to palliative care teams within their hospital or practice, yet only $21 \%$ of respondents report participation in palliative care rounds. Participation in palliative care rounds represents an opportunity for early identification of patients who would benefit from palliative radiation. Likewise, radiation oncology departments may run palliative care rounds specific to their patients who are receiving treatment and have a palliative care specialist present for input and or potential referral. Earlier access to palliative radiation therapy improves completion rates, reduces need for emergent inpatient consults, and increases outpatient palliative radiation treatments. ${ }^{28}$ Additionally, patients with advanced cancer have multidimensional physical, psychological, and spiritual pain. These patients would benefit from the expertise of a dedicated palliative radiation service that has advanced training in both PSC and radiation oncology, and dedicated palliative care support including a palliative care nurse and social worker.

An important limitation of this study is the selection bias inherent to any electronic survey of this nature, in which the views of those who chose to respond may not be generalizable to all radiation oncologists in the United States. It should be noted that our response rate of $16 \%$ is not unusual for voluntary electronic surveys or previously published ASTRO surveys. ${ }^{29-31}$ Our response rate was slightly lower than the $18.9 \%$ average response rate for ASTRO annual membership survey from 2013-2015, which offered participants an opportunity to win free registration to the annual ASTRO meeting (E. Wilson, oral communication, 2016). The variety of demographics encompassed by our participants does suggest a relatively representative sample, or at least that this topic is relevant to a variety of radiation oncologists, providing some reassurance of the validity of our findings. Furthermore, the survey was a self-assessment of knowledge and skills without an objective testing component. Physicians may overestimate their perceived knowledge, resulting in aboveaverage responses. ${ }^{32}$ Future research involving case-based scenarios and direct observation would provide more objective insight on knowledge and practice, respectively. Regardless, the survey is clinically meaningful in helping to direct the education efforts of ASTRO and other national organizations to fill a perceived knowledge gap.

\section{Conclusions}

This study indicates that most (91\%) radiation oncologists believe PSC is an important clinical competency within their profession. Respondents reported greater confidence in their ability to assess and manage pain than in their ability to manage depression, anxiety, anorexia, and fatigue. Despite this gap in knowledge, $42 \%$ of physicians have received no additional PSC education since completing their residency, and only $21 \%$ participate in palliative care patient rounds. There is a need for increasing educational efforts in PSC for practicing radiation oncologists and strengthening PSC training in residency programs. 
Increasing collaborations with other medical societies may improve communication and workflow to share in PSC responsibilities.

\section{Supplementary Material}

Refer to Web version on PubMed Central for supplementary material.

\section{Acknowledgments}

We would like to thank Stephanie Stevens, Emily Wilson, Liz Gardener, Dr. Margarita Racsa, Dr. Ted Deweese, Dr. Steve Lutz, Dr. Solomon Liao, and Dr. Kathryn Osann.

\section{References}

1. Lupu D, American Academy of Hospice and Palliative Medicine Workforce Task Force. Estimate of current hospice and palliative medicine physician workforce shortage. J Pain Symptom Manage. 2010; 40:899-911. [PubMed: 21145468]

2. Quill TE, Abernethy AP. Generalist plus specialist palliative care-Creating a more sustainable model. N Engl J Med. 2013; 368:1173-1175. [PubMed: 23465068]

3. Coia LR. Palliative radiation therapy in the United States. Can J Oncol. 1996; 6(Suppl 1):62-68. discussion 84. [PubMed: 8853540]

4. Murphy JD, Nelson LM, Chang DT, Mell LK, Le QT. Patterns of care in palliative radiotherapy: A population-based study. J Oncol Pract. 2013; 9:e220-e227. [PubMed: 23943892]

5. Janjan NA. An emerging respect for palliative care in radiation oncology. JPalliat Med. 1998; 1:8388. [PubMed: 15859875]

6. Frick E, Tyroller M, Panzer M. Anxiety, depression and quality of life of cancer patients undergoing radiation therapy: A cross-sectional study in a community hospital outpatient centre. Eur J Cancer Care (Engl). 2007; 16:130-136. [PubMed: 17371421]

7. Hahn CA, Dunn R, Halperin EC. Routine screening for depression in radiation oncology patients. Am J Clin Oncol. 2004; 27:497-499. [PubMed: 15596919]

8. Stasi R, Abriani L, Beccaglia P, et al. Cancer-related fatigue: Evolving concepts in evaluation and treatment. Cancer. 2003; 98:1786-1801. [PubMed: 14584059]

9. Temel JS, Greer JA, Muzikansky A, et al. Early palliative care for patients with metastatic nonsmall-cell lung cancer. N Engl J Med. 2010; 363:733-742. [PubMed: 20818875]

10. Rasca, M., Jones, JA., Dharmarajan, KV., et al. Palliative care training in radiation oncology: A national survey. American Society of Radiation Oncology: Annual Meetings; Boston, MA. 2016.

11. Snyder S, Hazelett S, Allen K, et al. Physician knowledge, attitude, and experience with advance care planning, palliative care, and hospice: Results of a primary care survey. Am JHosp Palliat Care. 2013; 30:419-424. [PubMed: 22798634]

12. Davies B, Sehring SA, Partridge JC, et al. Barriers to palliative care for children: Perceptions of pediatric health care providers. Pediatrics. 2008; 121:282-288. [PubMed: 18245419]

13. Oneschuk D, Fainsinger R, Hanson J, et al. Assessment and knowledge in palliative care in second year family medicine residents. J Pain Symptom Manag. 1997; 14:265-273.

14. Hilden JM, Emanuel EJ, Fairclough DL, et al. Attitudes and practices among pediatric oncologists regarding end-of-life care: Results of the 1998 american society of clinical oncology survey. J Clin Oncol. 2001; 19:205-212. [PubMed: 11134214]

15. Larrieux G, Wachi BI, Miura JT, et al. Palliative care training in surgical oncology and hepatobiliary fellowships: A national survey of program directors. Ann Surg Oncol. 2015; 22(Suppl 3):1181-1186.

16. Thomas RA, Curley B, Wen S, Zhang J, Abraham J, Moss AH. Palliative care training during fellowship: A national survey of U.S. Hematology and oncology fellows. J Palliat Med. 2015; 18:747-751. [PubMed: 26098204] 
17. Amini A, Miura JT, Larrieux G, et al. Palliative care training in surgical oncology and hepatobiliary fellowships: A national survey of the fellows. Ann Surg Oncol. 2015; 22:1761-1767. [PubMed: 25380685]

18. Buss MK, Lessen DS, Sullivan AM, Von Roenn J, Arnold RM, Block SD. Hematology/oncology fellows' training in palliative care: Results of a national survey. Cancer. 2011; 117:4304-4311. [PubMed: 21365618]

19. McDonald R, Chow E, Rowbottom L, et al. Quality of life after palliative radiotherapy in bone metastases: A literature review. J Bone Oncol. 2015; 4:24-31. [PubMed: 26579481]

20. Vuong S, Pulenzas N, DeAngelis $C$, et al. Inadequate pain management in cancer patients attending an outpatient palliative radiotherapy clinic. Support Care Cancer. 2016; 24:887-892. [PubMed: 26209950]

21. Janjan NA, Payne R, Gillis T, et al. Presenting symptoms in patients referred to a multidisciplinary clinic for bone metastases. J Pain Symptom Manag. 1998; 16:171-178.

22. Mercadante S. Opioid rotation for cancer pain: Rationale and clinical aspects. Cancer. 1999; 86:1856-1866. [PubMed: 10547561]

23. Smith EM, Gomm SA, Dickens CM. Assessing the independent contribution to quality oflife from anxiety and depression in patients with advanced cancer. Palliat Med. 2003; 17:509-513. [PubMed: 14526884]

24. Blouin G, Fowler BC, Dahlin C. The national agenda for quality palliative care: Promoting the national consensus project's domain of physical care and the national quality forum's preferred practices for physical aspects of care. J Pain Palliat Care Pharmacother. 2008; 22:206-212. [PubMed: 19042850]

25. Smith TJ, Temin S, Alesi ER, et al. American society of clinical oncology provisional clinical opinion: The integration of palliative care into standard oncology care. J Clin Oncol. 2012; 30:880-887. [PubMed: 22312101]

26. Davis D, O'Brien MA, Freemantle N, et al. Impact of formal continuing medical education: Do conferences, workshops, rounds, and other traditional continuing education activities change physician behavior or health care outcomes? JAMA. 1999; 282:867-874. [PubMed: 10478694]

27. Centers for Medicare and Medicaid Services. Medicare program: Revisions to payment policies under the physician fee schedule and revisions to Part B for CY 2016. FedReg. Nov 16.2015 80:20866.

28. Chang S, Smith CB, Morrison RS, Rosenzweig K, Dharmarajan KV. Icahn School of Medicine at Mount Sinai, New York, NY; Mount Sinai Medical Center, New York, NY. A palliative radiation oncology consult service's impact on care of advanced cancer patients with symptomatic bone metastases. J Clin Oncol. 2015; 33(Suppl 29S)

29. Eysenbach G. Improving the quality of web surveys: The checklist for reporting results of internet e-surveys (cherries). J Med Internet Res. 2004; 6:e34. [PubMed: 15471760]

30. Koontz BF, Benda R, De Los Santos J, et al. US radiation oncology practice patterns for posttreatment survivor care. Pract Radiat Oncol. 2016; 6:50-56. [PubMed: 26603597]

31. Nabavizadeh N, Elliott DA, Chen Y, et al. Image guided radiation therapy (IGRT) practice patterns and igrt's impact on workflow and treatment planning: Results from a national survey of American Society for Radiation Oncology members. Int J Radiat Oncol Biol Phys. 2016; 94:850-857. [PubMed: 26972658]

32. Dunning D, Heath C, Suls JM. Flawed self-assessment: Implications for health, education, and the workplace. Psychol Sci Public Interest. 2004; 5:69-106. [PubMed: 26158995] 


\section{NOTICE WARNING CONCERNING COPYRIGHT RESTRICTIONS}

The copyright law of the United States [Title 17, United States Code] governs the making of photocopies or other reproductions of copyrighted material. Under certain conditions specified in the law, libraries and archives are authorized to furnish a photocopy or other reproduction. One of these specified conditions is that the reproduction is not to be used for any purpose other than private study, scholarship, or research. If a user makes a request for, or later uses, a photocopy or reproduction for purposes in excess of "fair use," that use may be liable for copyright infringement. This institution reserves the right to refuse to accept a copying order if, in its judgement, fulfillment of the order would involve violation of copyright law. No further reproduction and distribution of this copy is permitted by transmission or any other means. 


\section{Table 1}

\section{Demographics of survey respondents}

\begin{tabular}{llr}
\hline Category & Characteristic & \multicolumn{1}{c}{ No. (\%) } \\
\hline Gender & Male & $433(67 \%)$ \\
& Female & $214(33 \%)$ \\
& Unknown & $2(<1 \%)$ \\
Years since residency & $0-5$ & $140(22 \%)$ \\
& $6-10$ & $118(18 \%)$ \\
& $11-20$ & $168(26 \%)$ \\
& 21 & $221(34 \%)$ \\
& Other/unknown & $2(<1 \%)$ \\
& Northeast & $179(28 \%)$ \\
& Southeast & $145(22 \%)$ \\
& Midwest & $140(21 \%)$ \\
& West & $102(16 \%)$ \\
& Southwest & $77(12 \%)$ \\
& Other/unknown & $6(1 \%)$ \\
& Private/community & $334(52 \%)$ \\
& Academic & $289(45 \%)$ \\
& Other/unknown & $19(3 \%)$ \\
\hline
\end{tabular}

Pract Radiat Oncol. Author manuscript; available in PMC 2018 March 01. 


\section{Table 2}

Percentages and frequencies of academic and community respondents stating they are "Moderately confident" or "Very confident" in their ability to care for various aspects of pain symptoms

\begin{tabular}{lcccc}
\hline & All respondents & Academic respondents & Community respondents & $\boldsymbol{P}$ value \\
\hline Assess pain & $95.7 \%(604)$ & $95.0 \%(267)$ & $96.0 \%(316)$ & .537 \\
Manage somatic pain (eg, bone pain) & $91.6 \%(578)$ & $89.7 \%(252)$ & $93.3 \%(307)$ & .106 \\
Titrate opioids (short and long acting) & $77.2 \%(487)$ & $74.4 \%(209)$ & $79.6 \%(262)$ & .123 \\
Manage neuropathic pain (eg, nerve root compression) & $69.1 \%(436)$ & $66.2 \%(186)$ & $71.7 \%(236)$ & .140 \\
Manage opioid related side effects & $68.5 \%(432)$ & $65.8 \%(185)$ & $70.2 \%(231)$ & .247 \\
$\begin{array}{l}\text { Rotate opioids (change from one type of opioid to } \\
\text { another) }\end{array}$ & $56.4 \%(356)$ & $50.5 \%(142)$ & $61.1 \%(201)$ & .009 \\
\hline
\end{tabular}


Table 3

Percentages and frequencies of academic and community respondents stating they are "Moderately confident" or "Very confident" in their ability to care for other non-pain symptoms

\begin{tabular}{lcccc}
\hline & All respondents & Academic respondents & Community respondents & $\boldsymbol{P}$ value \\
\hline $\begin{array}{l}\text { Manage other GI symptoms (dysphagia, diarrhea, } \\
\text { constipation) }\end{array}$ & $82.8 \%(520)$ & $77.5 \%(217)$ & $86.5 \%(283)$ & .004 \\
Manage nausea and vomiting & $82.3 \%(517)$ & $77.9 \%(218)$ & $85.9 \%(281)$ & .010 \\
Manage respiratory symptoms (cough, dyspnea) & $60.7 \%(381)$ & $56.4 \%(158)$ & $63.9 \%(209)$ & .060 \\
Manage insomnia & $44.3 \%(278)$ & $41.4 \%(115)$ & $47.4 \%(155)$ & .118 \\
Manage anorexia & $43.6 \%(274)$ & $40.0 \%(112)$ & $47.7 \%(156)$ & .057 \\
Manage fatigue & $40.6 \%(255)$ & $38.6 \%(108)$ & $42.5 \%(139)$ & .325 \\
\hline
\end{tabular}

GI, gastrointestinal. 


\section{Table 4}

Percentages and frequencies of academic and community respondents stating they are "Moderately confident" or "Very confident" in their ability to perform psychological, cultural, and spiritual aspects of care

\begin{tabular}{|c|c|c|c|c|}
\hline & All respondents & Academic respondents & Community respondents & $P$ value \\
\hline $\begin{array}{l}\text { Recognize when to incorporate interpreter services } \\
\text { when caring for a patient/family with advanced cancer }\end{array}$ & $84.2 \%(523)$ & $83.5 \%(233)$ & $85.7 \%(275)$ & .464 \\
\hline $\begin{array}{l}\text { Recognize the importance of culture with respect to } \\
\text { patient/family values, preferences, and goals }\end{array}$ & $80.4 \%$ (499) & $80.3 \%$ (224) & $81.0 \%(260)$ & .826 \\
\hline Recognize when to refer to mental health professionals & $74.9 \%(465)$ & $74.2 \%(207)$ & $75.7 \%(243)$ & .671 \\
\hline $\begin{array}{l}\text { Assess and recognize the role of spirituality and } \\
\text { spiritual issues within illness }\end{array}$ & $68.1 \%(423)$ & $64.9 \%(181)$ & $71.3 \%(229)$ & .069 \\
\hline $\begin{array}{l}\text { Recognize when to refer to spiritual care professionals } \\
\text { (eg, chaplain, clergy) }\end{array}$ & $59.6 \%(370)$ & $54.5 \%(152)$ & $64.8 \%(208)$ & .010 \\
\hline Assess psychosocial issues & $52.2 \%(324)$ & $49.5 \%(138)$ & $53.9 \%(173)$ & .279 \\
\hline Initial management of anxiety & $49.3 \%(306)$ & $45.2 \%(126)$ & $53.9 \%(173)$ & .033 \\
\hline Initial management of depression & $33.7 \%(209)$ & $28.3 \%(79)$ & $38.3 \%(123)$ & .010 \\
\hline
\end{tabular}




\section{Table 5}

Percentages and frequencies of academic and community respondents stating they are "Moderately confident" or "Very confident" in their ability to perform social, ethical, and legal aspects of care

\begin{tabular}{|c|c|c|c|c|}
\hline & All respondents & Academic respondents & Community respondents & $P$ value \\
\hline Discuss transition from curative to palliative care & $82.8 \%(511)$ & $80.1 \%(221)$ & $85.0 \%(272)$ & .113 \\
\hline $\begin{array}{l}\text { Collaborate with interdisciplinary team to develop a } \\
\text { palliative care plan }\end{array}$ & $76.0 \%(469)$ & $76.4 \%(211)$ & $75.9 \%(243)$ & .884 \\
\hline Lead/participate in a family meeting & $70.8 \%(437)$ & $69.2 \%(191)$ & $71.3 \%(228)$ & .585 \\
\hline Discuss eligibility criteria and benefits of hospice & $67.9 \%(419)$ & $62.0 \%(171)$ & $73.4 \%(235)$ & .003 \\
\hline Assess patient decision-making capacity & $67.6 \%(417)$ & $63.0 \%(174)$ & $71.3 \%(228)$ & .033 \\
\hline $\begin{array}{l}\text { Recognize when to refer for bereavement services/ } \\
\text { resources }\end{array}$ & $57.7 \%(356)$ & $55.4 \%(153)$ & $60.3 \%(193)$ & .228 \\
\hline $\begin{array}{l}\text { Manage conflict when there is disagreement between } \\
\text { the patient/family and the clinical team }\end{array}$ & $52.8 \%(326)$ & $50.4 \%(139)$ & $54.1 \%(173)$ & .367 \\
\hline
\end{tabular}




\section{Table 6}

Percentages and frequencies of academic and community respondents stating they are "Moderately confident" or "Very confident" in their ability to perform various aspects of advance care planning

\begin{tabular}{|c|c|c|c|c|}
\hline & All respondents & Academic respondents & Community respondents & $P$ value \\
\hline Communicate "bad news" with honesty and compassion & $92.1 \%(563)$ & $90.9 \%(249)$ & $93.7 \%(296)$ & .202 \\
\hline Discuss prognosis with patient/family & $85.4 \%(522)$ & $83.6 \%(229)$ & $87.0 \%(275)$ & .236 \\
\hline $\begin{array}{l}\text { Discuss and incorporate patient/family values, } \\
\text { preferences, and goals into palliative care plan }\end{array}$ & $82.3 \%(503)$ & $79.2 \%(217)$ & $84.8 \%(268)$ & .075 \\
\hline $\begin{array}{l}\text { Respond to strong patient emotions (eg, fear, anger, } \\
\text { sadness) }\end{array}$ & $81.3 \%(497)$ & $78.8 \%(216)$ & $83.2 \%(263)$ & .173 \\
\hline Estimate a patient's prognosis & $65.8 \%(402)$ & $59.5 \%(163)$ & $71.5 \%(226)$ & .002 \\
\hline Discuss "do-not-resuscitate" orders with patient/family & $65.1 \%(398)$ & $64.6 \%(177)$ & $65.8 \%(208)$ & .755 \\
\hline $\begin{array}{l}\text { Discuss preparation of a living will or selection of a } \\
\text { health care proxy with patient/family }\end{array}$ & $56.5 \%(345)$ & $56.2 \%(154)$ & $56.6 \%(179)$ & .914 \\
\hline
\end{tabular}

